53 ATTITUDES AND PERCEPTIONS OF PATIENTS WITH PULMONARY FIBROSIS AND THEIR CARERS TOWARDS USE OF OXYGEN THERAPY: A RAPID REVIEW

Mala Mann, Amanda Woodward, Anthony Byrne. Cardiff University, UK

10.1136/spcare-2019-mariecuriepalliativecare.53

Background Patients with Idiopathic Pulmonary Fibrosis (IPF) develop progressive respiratory failure and eventually require supplementary oxygen therapy. However, circumstantial evidence suggests some patients are reluctant to use such treatment. This can be a barrier to providing high quality palliative/supportive care.

Aim To identify attitudes and perceptions of patients with pulmonary fibrosis and their carers towards use of oxygen therapy.

Methods Key databases were searched for papers published between 2008-2018. Supplementary searches were also conducted. We followed Palliative Care Evidence Review Service (PaCERS) methodology ${ }^{1}$, developed to conduct rapid reviews requested by clinicians/clinical services.

Results Seven studies met inclusion criteria. Consistent themes included avoidance due to stigma, negative perceptions of prognostic implications, loss of role and social functioning, managing the practicalities, and equipment malfunction.

Conclusion The findings have important implications for multidisciplinary team care prior to and following the introduction of supplemental oxygen, particularly in relation to patient and carer preparedness and education, practicalities of equipment use and the particular needs of carers. Introduction of oxygen should act as a trigger for considering specialist palliative care review.

\section{BARRIERS TO ACCESSING AND DELIVERING HOSPICE CARE SERVICES FOR DIVERSE COMMUNITIES: A RAPID REVIEW}

Ishrat Islam, Elin Baddeley, Delyth Morris, Annmarie Nelson, Stephanie Sivell. Cardiff University, UK

\subsection{6/spcare-2019-mariecuriepalliativecare.54}

Introduction Diverse communities - people with dementia, intellectual disabilities (ID) and religious beliefs, have lower rates in accessing palliative and hospice care services (Johnson, 2013). Healthcare providers also encounter problems in delivering appropriate services to these communities (Koffman, 2014).

Aim To identify the barriers to accessing and delivering hospice care services for diverse communities.

Method A rapid review was conducted using a systematic search protocol. The search yielded 95 relevant titles from which 34 were screened for data extraction and 23 met the inclusion criteria. Thematic approach was used for data analysis.

Results The overall barriers identified were mainly physical, psycho-social and economic. People with dementia were challenged by the uncertainty of their survival time. Their lack of awareness of service facilities, availability and financial circumstances hindered them accessing hospice care. Difficulties associated with prognostication; communication; inadequate medical interventions; and appropriateness of palliative care intervention were the main barriers for those providing palliative care to this group. For individuals with ID, social, emotional and cognitive issues (patient understanding, capacity to consent etc.) were the main factors affecting palliative care provision. The main barriers for services providers were lack of guidelines and support, access to general healthcare facilities and internal staffing issues. For those with specific religious beliefs, unwillingness to involve outsiders, being well supported by their religious community, distrust of medical establishments and unawareness of the need and availability of services were the main barriers. Lack of diversity among health care staff and resource constraints were barriers to service delivery.

Conclusions Addressing specific needs of diverse communities would encourage better utilisation of hospice care.

\section{5 'TILL DEATH US DO PART' AN EXPLORATION OF THE PSYCHOLOGICAL IMPLICATIONS WHEN CARING FOR A LOVED ONE AT END OF LIFE}

Andrea Ward. Marie Curie, UK

\subsection{6/spcare-2019-mariecuriepalliativecare.55}

Background To die in their own home is one of the key elements of care for approximately $70 \%$ of people living with a terminal illness, with the care predominantly provided at end of life by family caregivers including spouses (Patel 2016). Family members often care for their relative at this stage of life for long periods of time untrained and alone resulting in acute hospice and hospital admissions and an unfilled wish (McGrath 2016).

Aim Review Question: What are the psychological implications of caring for a loved one at end of life in the home setting?

Review objectives:

- To explore any effect on personal relationships.

Method A qualitative systematic search for articles was performed in February 2017 with 757 primary research papers identified from the searches, assessed against the criteria and following analysis were narrowed down to nine.

Results Following thematic analysis, the psychological implications of caring were grouped into five distinct categories and subsequent themes as follows 1) Isolation 2) Responsibility 3) Guilt 4) Interdependency 5) Stress. Effect on relationships identified two themes: Stretched limits, and obligation.

Conclusion The review did demonstrate how caring for a dying family member in the home setting does have psychological implications on the carer and within the relationship with their loved one. Caring for a relative does require a level of resilience and psychological strength to enable this level of caregiving due to the limitations on the carer's own life. The involvement of the health professional is imperative to ensure these families are able to maintain relationships and fulfil their wishes safely. 